\title{
On the use of a nickel-tube resonator for measuring the complex shear modulus of liquids in the $\mathrm{kHz}$-range
}

\author{
H. A. Waterman, M. Oosterbroek, G. J. Beukema, and E. G. Altena
}

With 4 figures and 2 tables

(Received February 2, 1979)

\begin{tabular}{|c|c|}
\hline Notations & \\
\hline & outer and inner radii of the tube \\
\hline$B_{\sqrt{2}}, B_{\sqrt{2}}^{0}$ & $\begin{array}{l}3 \mathrm{~dB} \text { band-width of resonance curve of the } \\
\text { loaded and unloaded tube }\end{array}$ \\
\hline$c, c_{0}$ & $\begin{array}{l}\text { phase velocities of torsional waves in the } \\
\text { loaded and unloaded tube }\end{array}$ \\
\hline$c^{*}, c_{0}^{*}$ & $\begin{array}{l}\text { complex velocities of propagation of tor- } \\
\text { sional waves in the loaded and unloaded tube }\end{array}$ \\
\hline$c^{*}$ & $=c_{1}+i c_{2}$ \\
\hline$c_{L}^{*}$ & $\begin{array}{l}\text { complex velocity of propagation of shear } \\
\text { waves in the fluid }\end{array}$ \\
\hline$f$ & frequency \\
\hline$f_{n}, f_{n}^{0}$ & $\begin{array}{l}\text { resonance frequency of } n \text {-th overtone of the } \\
\text { loaded and unloaded tube }\end{array}$ \\
\hline$G^{*}$ & complex shear modulus of the tube material \\
\hline$G_{L}^{*}$ & complex shear modulus of the fluid \\
\hline$i$ & $=\sqrt{-1}$ \\
\hline$I_{0}$ & moment of inertia per $\mathrm{cm}$ of unloaded tube \\
\hline$I$ & $\begin{array}{l}\text { effective moment of inertia per } \mathrm{cm} \text { of loaded } \\
\text { tube }\end{array}$ \\
\hline$K$ & instrument constant \\
\hline$l$ & length of the tube \\
\hline$m$ & $=\bar{b} / a$ \\
\hline$M$ & angular momentum \\
\hline$n$ & positive integer \\
\hline$R_{L}, X_{L}$ & $\begin{array}{l}\text { real and imaginary parts of the characteristic } \\
\text { plane shear impedance of the fluid }\end{array}$ \\
\hline$Z_{L}$ & $\begin{array}{l}\text { characteristic plane shear impedance of the } \\
\text { fluid }\left(=R_{L}+i X_{L}\right)\end{array}$ \\
\hline$Z_{\mathrm{cy} 1}$ & $\begin{array}{l}\text { characteristic cylindrical shear impedance of } \\
\text { the fluid }\end{array}$ \\
\hline$\alpha, \alpha_{0}$ & $\begin{array}{l}\text { damping factors of torsional waves in the } \\
\text { loaded and unloaded tube }\end{array}$ \\
\hline$\beta, \beta_{0}$ & $\begin{array}{l}\text { phase factors of torsional waves in the loaded } \\
\text { and unloaded tube }\end{array}$ \\
\hline$\Gamma$ & propagation constant $(=\alpha+i \beta)$ \\
\hline$\delta$ & loss angle \\
\hline$\zeta$ & $\equiv \tan \cos ^{-1}\left(\xi / \xi_{\max }\right)$ \\
\hline$\eta$ & viscosity \\
\hline$\dot{\theta}$ & angular displacement \\
\hline$\lambda$ & wave length \\
\hline$\xi$ & displacement amplitude \\
\hline$\rho, \rho_{0}$ & densities of the fluid and the tube material \\
\hline$\omega$ & angular frequency $(=2 \pi f)$ \\
\hline$\omega_{n}^{0}$ & $=2 \pi f_{n}^{0}$ \\
\hline$\omega_{R}$ & reduced frequency \\
\hline
\end{tabular}

\section{Introduction}

In 1968 Glover et al. (1) introduced an instrument for measuring liquid shear impedances in the frequency range $20-100 \mathrm{kHz}$. Torsional oscillations are generated magnetostrictively in a thin-walled Ni-tube and travel along this tube, showing an attenuation of $\alpha$ Nepers per $\mathrm{cm}$ and a phase shift of $\beta$ radians per $\mathrm{cm}$. When the tube is immersed in a liquid, there is an extra attenuation of $\Delta \alpha$ Nepers per $\mathrm{cm}$ due to the radiation of energy into the liquid and a change of $\Delta \beta$ radians per $\mathrm{cm}$ due to the extra inertial loading of the tube. The authors showed that $\Delta \alpha$ and $\Delta \beta$ are proportional to $\left(1+m^{3}\right) /$ $\left(1-m^{4}\right)$. For a tube with an outer diameter of $0.4 \mathrm{~cm}$ and a wall thickness of $0.01 \mathrm{~cm}$ this factor is $\sim 9$. Hence for a given liquid the values of $\Delta \alpha$ and $\Delta \beta$ and therefore the sensitivity are enhanced by roughly an order of magnitude with respect to their values for a solid rod with the same diameter.

For the measurement of $\Delta \alpha$ and $\Delta \beta$ a pulse cancellation technique was used. However, as stated by the authors, "one difficulty has been the overlapping of the measuring pulses with small pulse reflections from the dry-tube/wettube boundary and also from the damping device". In principle this problem can be solved by arranging the geometry of the system in such a way that these small pulse reflections do not overlap any of the measuring pulses. From some preliminary measurements performed with a prototype apparatus built along these lines in our laboratory we discovered however that solving the problem in this way puts a severe constraint on the geometry, eventually leading to inconveniently long tubes. That is why for instance Cook and Matheson (2) used tubes 
with a length of $2 \mathrm{~m}$, which introduces considerable difficulties with thermostating.

More recently an apparatus was described by Nakajima et al. (3) in which a long rod of fused quartz is excited into torsional resonant oscillations by means of a quartz crystal. By measuring the decrease in resonant frequency and increase in decay rate when a liquid is applied around the rod the liquid shear impedance can be found. The method is used at the fundamental resonance and overtones in the frequency range $2-20 \mathrm{kHz}$.

Since the increased sensitivity offered by the use of a thin-walled tube has many advantages and, moreover, as we had hoped that higher overtones might be obtained, we decided to investigate whether an apparatus based on a magnetostrictively excited $\mathrm{Ni}$-tube resonator could be relazized.

\section{Theory}

An exact analytical solution for the propagation of torsinal waves along a tube immersed in a liquid has been given by Glover et al. (1) in terms of Bessel functions. For practical cases this solution can be simplified by approximating the Bessel functions by the leading terms in their series expansions. In this way a solution is found that can be handled by the experimentalist. From the formulae it is clear that with this approximation the motion corresponds to a rigid rotation of each cross-section of the tube around its centre, i.e. the same motion found from the exact solution valid for a tube in a vacuum (4). Therefore the approximation $(|k a| \ll 1)$ as used by Glover et al. (1) and by McSkimin (5) is equivalent to the assumption that cross-sections perform rigid rotations. It is obvious therefore to make this assumption at the beginning. Consider an element of the loaded tube with length $d z$, where $d z \ll \lambda$. In a mechanical sense this tube element is circuited in parallel (the particle velocities are continuous across the boundaries) with the liquid elements inside and outside the tube. Consequently the total mechanical impedance $Z$ equals the sum of the mechanical impedances of the elements. (In view of the movement we will use rotational impedances defined by $Z \equiv-M / \dot{\theta}$.) Therefore we have:

$Z=Z_{0}+Z_{1}+Z_{2}$.
In eq. [1] $Z_{0}$ is the impedance of the unloaded tube element, $Z_{1}$ and $Z_{2}$ are the impedances of the outer and inner fluid elements at the boudaries:

$Z_{0} \equiv-\frac{M}{\dot{\theta}}=-\frac{I_{0} \ddot{\theta}}{\ddot{\theta}}=-i \omega I_{0}$

where

$I_{0}=\frac{\pi}{2} \rho_{0} a^{4}\left(1-m^{4}\right)$

for $Z_{1}$ and $Z_{2}$ it is found:

$\left.\begin{array}{l}Z_{1}=-2 \pi a^{3} Z_{\mathrm{cyl}} \\ Z_{2}=-2 \pi b^{3} Z_{\mathrm{cyl}}\end{array}\right\}$

In eq. [4] $Z_{\text {cyl }}$ is the characteristic cylindrical shear impedance of the fluid, whereas the plane shear impedance $Z_{L}$ is required. $Z_{\text {cyl }}$ and $Z_{L}$ differ only by a small amount. Moreover it was shown (1) that the correction due to the outgoing wave from the outer surface of the tube almost cancels the correction due to the ingoing wave from the inner surface, provided $m \simeq 1$.

From eqs. [1]- [4] then follows:

$$
\begin{aligned}
Z & =-i \omega I_{0}\left\{1+\frac{2 \pi a^{3}\left(1+m^{3}\right) Z_{L}}{i \omega I_{0}}\right\} \\
& \equiv-i \omega I
\end{aligned}
$$

So in a formal sense the effect of the fluid can be described by an additional moment of inertia $\Delta I$ amounting to

$\Delta I=\frac{2 \pi a^{3}\left(1+m^{3}\right) Z_{L}}{i \omega}$

For the unloaded and loaded tube we therefore have:

$c_{0}^{*}=\left\{\frac{G^{*} a^{4}\left(1-m^{4}\right)}{2 I_{0}}\right\}^{1 / 2}$,
$c^{*}=\left\{\frac{G^{*} a^{4}\left(1-m^{4}\right)}{2 I}\right\}^{1 / 2}$.

With $c_{0}^{*}=i \omega / \Gamma_{0}$ and $c^{*}=i \omega / \Gamma$ it follows from eqs. [5]-[7]:

$\Gamma-\Gamma_{0}=(\Delta \alpha+i \Delta \beta)=\frac{2 Z_{L}}{a \rho_{0} c_{0}^{*}}\left(\frac{1+m^{3}}{1-m^{4}}\right)$,

with the approximation $\left(I_{0} / I\right)^{1 / 2} \simeq 1-\left(\Delta I / 2 I_{0}\right)$ used. For $c_{0}^{*} \simeq c_{0}$ eq. [8] passes into eq. [16] 
of Glover et al. (1). The resonance condition for a free-free tube is ${ }^{1}$ ):

$\beta l=n \pi \quad(n=1,2,3, \ldots)$,

with

$\beta=\frac{\omega}{c}$.

From [9] and [10] it is seen that in order to restore resonance when the tube is immersed from the air into a fluid, $\omega_{n}^{0}$ has to be adjusted so as to keep $\beta$ constant. From [10] we find:

$\frac{\Delta \omega}{\omega_{n}^{0}}=\frac{\Delta c}{c_{0}}=-1+\frac{c}{c_{0}}$

It was shown (6) that the phase velocity $c$ for plane waves can be written as

$c=c_{1}\left\{1+\left(\frac{\alpha}{\beta}\right)^{2}\right\}$

with

$c^{*}=c_{1}+i c_{2}$.

Further we have (6)

$\frac{c_{2}}{c_{1}}=\frac{\alpha}{\beta}$.

From eqs. [12] $-[14]$ it follows that

$c=c^{*}\left(1-i \frac{\alpha}{\beta}\right)$

and from eqs. [15], [11] and [17]

$$
\begin{aligned}
& \frac{\Delta \omega}{\omega_{n}^{0}}=-1 \\
& +\frac{\left(1-\frac{\Delta I}{2 I_{0}}\right)\left\{1+\frac{\alpha_{0}}{\beta_{0}} \cdot \frac{\alpha}{\beta}-i\left(\frac{\alpha}{\beta}-\frac{\alpha_{0}}{\beta_{0}}\right)\right\}}{1+\left(\frac{\alpha}{\beta}\right)^{2}}
\end{aligned}
$$

Here as well the approximation $\left(I_{0} / I\right)^{1 / 2} \simeq$ $1-\left(\Delta I / 2 I_{0}\right)$ has been used. For $\alpha / \beta \ll 1$ and $\Delta \omega / \omega_{0} \ll 1$ it finally follows from eqs. [16], [3] and $[6]$ that

$f_{n}^{0}-f_{n}=\frac{X_{L}}{\pi \rho_{0} a}\left(\frac{1+m^{3}}{1-m^{4}}\right)=K X_{L}$,

1) Strictly speaking, phase resonance coincides with velocity resonance only for a lumped resonator. For a not too high damping $\left(\alpha^{2} / \beta^{2} \ll 1\right.$, compare [19]), however, this also holds with good approximation for a distributed resonator.
$B_{\sqrt{2}}-B_{\sqrt{2}}^{0}=\frac{2 R_{L}}{\pi \rho_{0} a}\left(\frac{1+m^{3}}{1-m^{4}}\right)=2 K R_{L}$

where in deriving $[18]$ the relation

$\frac{\alpha}{\beta}=\pi \tan \frac{\delta}{2} \simeq \frac{\pi}{2} \tan \delta=\frac{\pi B_{\sqrt{2}}}{2 f_{n}}$

has been used.

In this calculation the small changes in amplitude and phase due to reflections at the end faces of the tube are neglected. It is easily shown, however, that even for high loading of the tube these changes have no measurable effect.

\section{Apparatus}

A prototype, as shown in figure 1, was built by using equipment readily available in our laboratory.

The Ni-tube is clamped in the middle with the aid of a teflon or steel ring. Before mounting it was magnetically polarized by passing a current of the order of $1000 \mathrm{~A}$ through it during $1 \mathrm{msec}$ from a bank of capacitors. In this way the tube is polarized circumferentially. It is set into torsional oscillation with the aid of a transmitting coil providing an alternating axial magnetic field which forces the tube to twist. The oscillations of the tube are detected by a receiving coil by means of the reverse effect. The tube is surrounded by a glass tube containing the liquid. This part of the apparatus, fastened to a frame, is immersed in a liquid thermostat with a temperature stability of $\sim 0.01^{\circ} \mathrm{C}$. The transmitting coil is fed from a synthesizer, while the signal delivered by the receiving coil is measured with an a.c. voltmeter. Both transducers can be adjusted in axial direction. Besides in the fundamental mode the tube can resonate in many overtones. In the fundamental mode the length of the tube equals half a wavelength, i. e. there is a node in the middle and antinodes at both ends. Due to the disturbance caused by the clamp only odd overtones can be used.

For the excitation of a particular overtone both the transmitting and the receiving coils have to be positioned at nodal planes for this overtone. In this way it was possible to excite a tube of $40 \mathrm{~cm}$ length up to its 51 st overtone. This limit is set by the resonance frequency of the detecting circuit and can probably be raised by lowering the capacitances of coil and leads. Since in the fundamental mode there is only a nodal plane in the middle of the tube, both transducers were positioned as close to each other as the clamping device permitted in order to measure this mode. In this position the distance between the centres of the coils is about $10 \mathrm{~cm}$. The clamping device is of a very simple construction, as schematically shown in figure 1 . The thickness of the clamping ring is $2 \mathrm{~mm}$, which is one order of magnitude smaller than the wave-length at the highest frequency used, viz. $\sim 15 \mathrm{~mm}$ at $200 \mathrm{kHz}$. Since the Ni-tube were not perfectly straight and the clearance between $\mathrm{Ni-tube}$ and glass tube is only 


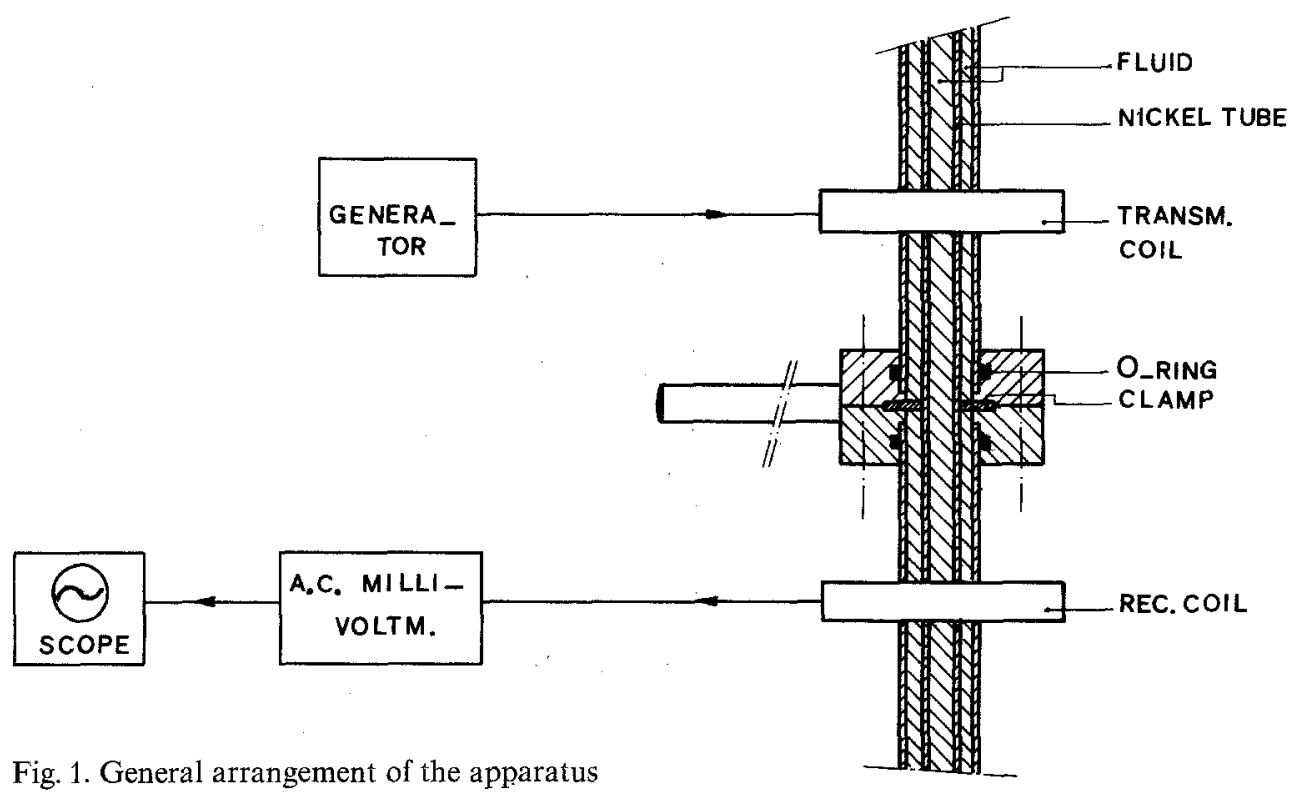

$1 \mathrm{~mm}$, some skill is required to position the tube firmly without touching the glass wall. The clearance was made as small as possible for two reasons, (i) reducing the amount of liquid required and (ii) increasing the homogeneity of the axial magnetic field.

The coils, made out of copper wire having a diameter of $0.1 \mathrm{~mm}$, were wound on a coil former of hard plastic. The number of turns of the transmitting and receiving coils are 50 and 500 respectively. They are embedded in pot-cores (Philips 36/22-3B7) from which the cores were removed, see figure 2 . Finally this assembly is mounted in a water-tight cylindrical housing.

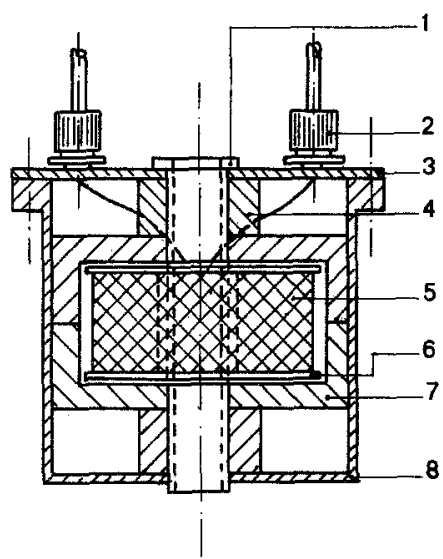

Fig. 2. Mechanical construction of the coil housings 1. tube of hard plastic, 2. feed-through for coaxial cable, 3. cover plate, 4. filling-in piece, 5. coil, 6. coil former, 7 . ferrite pot, 8 . housing

The capacitor bank consists of six "flash"-type electrolytic capacitors connected in parallel, each capacitor having a capacitance of $2500 \mu \mathrm{F}$ and a maximum voltage of $250 \mathrm{~V}$. For the discharge of the bank a thyristor is used as a switching device. In order to limit the tyristor current to a safe value each capacitor is equiped with a Kanthal-wire limiting resistor of $0.48 \Omega$. These resistors moreover provide a uniform discharge of the capacitors. A resistor $(1 \mathrm{~m} \Omega)$ is also connected in series with the tube in order to monitor the pulse on a storage oscilloscope.

\section{Measuring procedure and data handling}

In order to find the components $R_{L}$ and $X_{L}$ of the complex shear impedance from eqs. [17] and [18] the resonance frequencies of the tube in air and immersed in the sample, $f_{n}^{0}$ and $f_{n}$ resp., the $3 \mathrm{~dB}$ band-widths $B_{\sqrt{2}}^{0}$ and $B_{\sqrt{2}}$ of the resonance curves and $K$ have to be known. $K$ can be calculated from the geometry of the tube and its density. Alternatively $K$ can be found from measurements on a Newtonian liquid with known viscosity and density. The latter procedure is commonly used with the torsional-crystal apparatus $(7,8)$, where relations similar to our eqs. [17] and [18] appear. It turned out that this procedure did not result in an essential improvement of the measurements in this investigation, so the theoretical value of $K$ was used. To start with, the fundamental mode in air was measured with the coils as close to each other as possible. Subsequently the coils were separated in order to optimize the signal for measuring the third overtone. For the fifth and subsequently higher overtones more nodal planes become available. It was then found that the results are slightly dependent on the choice of the nodal planes used. Since the air meas- 
urements have to be related to measurements with the tube immersed in the sample the same nodal planes should be used in both measurements. After the air measurements are performed the glass tube is filled with the sample and the measuring procedure is repeated. The tube is filled with the aid of a hypodermic syringe brought in at the lower side in order to avoid trapped air bubbles, which render measurements impossible. In order to determine the resonance frequency and the $3 \mathrm{db}$ band-width from the measured data a transform according to Nederveen (9) is used. This author showed that under reasonable assumptions the equation for the resonance curve of a linear second-order system can be written in the form ${ }^{2}$ )

$\zeta \equiv \tan \cos ^{-1}\left(\frac{\xi}{\xi_{\max }}\right)=\frac{2\left|f-f_{n}\right|}{B_{\sqrt{2}}}$.

Here $\xi$ is the amplitude at angular frequency $\omega$ and $\xi_{\max }$ the amplitude at resonance. Hence a plot of $\zeta$ versus $f$ should be a straight line which crosses the $f$-axis at $f=f_{n}$, whereas its slope is determined by $B_{\sqrt{2}}$. For finding out how well the data fit this line a linear regression analysis is performed $(10,11)$. The assumptions made in deriving eq. [20] are that $M_{1}$ and $M_{2}$, the real and imaginary parts of the relevant elastic modulus, are constant over the limited

\footnotetext{
$\left.{ }^{2}\right) \mathrm{Eq}$ [20] is derived for the displacement of a lumped resonator. It can be shown, however, that this equation is also applicable for the displacement velocity of a resonator with distributed mass and stiffness (12).
}

frequency range of the measurements and moreover $f_{n} \gg B_{\sqrt{2}}$.

\section{Measuring results}

In order to test the apparatus measurements were performed on various Newtonian liquids and a solution of polystyrene in a Newtonian solvent. The Ni-tube used had a length of about $40 \mathrm{~cm}$, an outside diameter of $0.4 \mathrm{~cm}$ and a wall thickness of $0.01 \mathrm{~cm}$. From the measured inner and outer diameters of the tube and its density the factor $K$ in eqs. [17] and [18] was estimated to be $(1.88 \pm 0.04) \mathrm{g}^{-1} \mathrm{~cm}^{2}$. Actually the value $K=1.84 \mathrm{~g}^{-1} \mathrm{~cm}^{2}$ best fitted the results and this value was used.

In table 1 the values of $R_{L}$ and $X_{L}$ calculated from a number of measured resonance curves are summarized for three Newtonian liquids, together with their theoretical values.

In performing the two series of measurements on dibutylphthalate (D.B.P.) the resonance curves of the unloaded tube were first of all measured for a number of overtones in one run and thereafter the resonance curves of the loaded tube in a second run.

From table 1 it is seen that there is a reasonable agreement between measured and theoretical values of $R_{L}$, but for $X_{L}$ there are some substantial deviations. These deviations are far outside the quoted uncertainty ranges as derived from linear regression analysis of the measured data according to eq. [20]. Therefore systematic errors must be responsible for these large deviations. For illustrating this, in

Table 1

\begin{tabular}{|c|c|c|c|c|c|}
\hline Sample & $\begin{array}{c}\text { Viscosity } \\
{[\mathrm{cP}]}\end{array}$ & $\begin{array}{c}\text { Frequency } \\
{[\mathrm{Hz}]}\end{array}$ & $\begin{array}{c}R_{L} \\
{[\text { dyne s cm }} \\
-3]\end{array}$ & $\begin{array}{c}X_{L} \\
{[\text { dyne s cm}} \\
-3\end{array}$ & $\begin{array}{c}(\pi \rho \eta f)^{1 / 2} \\
{\left[\text { dyne } \mathrm{cm}^{-3}\right]}\end{array}$ \\
\hline dist. water $\left(20.6^{\circ} \mathrm{C}\right)$ & 0.998 & 3367 & 10.2 & 11.3 & 10.2 \\
\hline hexadecane $\left(20.6^{\circ} \mathrm{C}\right)$ & 3.06 & 3365 & 16.0 & 16.2 & 16.5 \\
\hline D.B.P. $\left(46.1^{\circ} \mathrm{C}\right)$ & 7.62 & $\begin{array}{r}3634 \\
11014 \\
18352 \\
33092 \\
40471 \\
47860 \\
92124 \\
188196\end{array}$ & $\begin{array}{l}29.5 \\
53 \\
67 \\
94 \\
100 \\
110( \pm 1) \\
150( \pm 2) \\
218( \pm 5)\end{array}$ & $\begin{array}{l}29.6 \\
53 \\
67 \\
94 \\
98 \\
133( \pm 1) \\
163( \pm 1) \\
227( \pm 2)\end{array}$ & $\begin{array}{l}29.9 \\
52 \\
67 \\
91 \\
100 \\
109 \\
151 \\
215\end{array}$ \\
\hline D.B.P. $\left(81.4^{\circ} \mathrm{C}\right)$ & 3.19 & $\begin{array}{r}4030 \\
12149 \\
20259\end{array}$ & $\begin{array}{l}20.1 \\
33.3 \\
47.2\end{array}$ & $\begin{array}{l}19.7 \\
33.6 \\
44.2\end{array}$ & $\begin{array}{l}20.0 \\
34.8 \\
45.0\end{array}$ \\
\hline
\end{tabular}



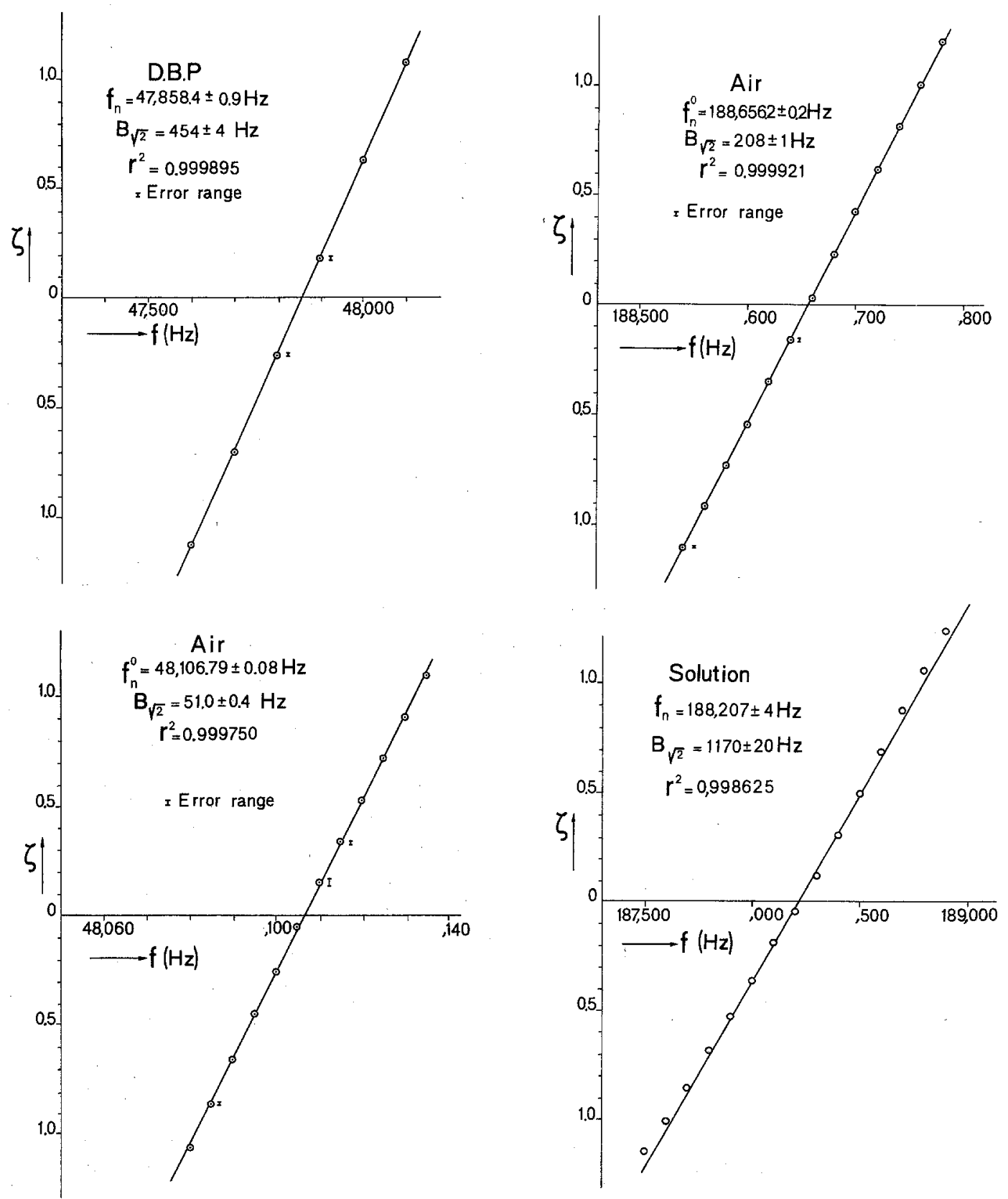

Fig. 3. Plot of the resonance curve, according to eq. [20], of the 13 th overtone of a Ni-tube resonator, about $40 \mathrm{~cm}$ long, (a) immersed in D.B.P., (b) in air

figures $3 a$ and $3 b$ the data for the loaded and unloaded tube for one of these overtones are plotted according to eq. [20]. We will come back to this point in the discussion at the end of this paper. In figures $3 a$ and $3 b$ the uncertainty ranges due to the reading error of the a.c. voltmeter are also drawn for various measuring points. It turns out that the a.c. voltmeter puts a limit to the accuracy of the measurements. It

Fig. 4. Plot of the resonance curve, according to eq. [20], of the 51 th overtone of a $\mathrm{Ni}$-tube resonator (a) in air, (b) immersed in a solution of polystyrene in D.B.P.

is rather surprising that this is even the case for the resonance curve of the unloaded tube at the higest overtone used, see figure $4 \mathrm{a}$, although at this frequency the length of the coils exceeds the wave-length in the tube. For high loading of the tube at this frequency significant deviations from a straight line occur due to the vicinity of neighbouring overtones, see figure $4 \mathrm{~b}$. As a consequence of this mode coupling 
only a small part of the resonance curve can be used for analysis, which restricts the accuracy.

As an example of a visco-elastic fluid we performed various measurements on a solution of polystyrene in D.B.P. Data on the composition of this solution are given below ${ }^{3}$ ).

Table 2

\begin{tabular}{ccccc}
\hline $\log \omega_{R}$ & $R_{L}\left[\right.$ dyne s cm $\left.^{-3}\right]$ & \multicolumn{2}{c}{$X_{L}[$ dyne s cm } \\
\hline 0.523 & $\left.50^{\mathrm{a}}\right)$ & $\left.52^{\mathrm{b}}\right)$ & $\left.38^{\mathrm{a}}\right)$ & $\left.37^{\mathrm{b}}\right)$ \\
0.601 & 55 & 56 & 42 & 41 \\
0.824 & 76 & 71 & 52 & 53 \\
1.006 & 80 & 80 & 61 & 61 \\
1.228 & 95 & 98 & 76 & 75 \\
1.375 & 110 & 112 & 91 & 87 \\
1.484 & 117 & 122 & 97 & 98 \\
1.664 & 141 & 145 & 135 & 120 \\
1.930 & 189 & 188 & 166 & 162 \\
2.239 & 260 & 257 & 237 & 229 \\
\hline
\end{tabular}

a) Results of this investigation.

b) Data obtained with torsional quartz crystal apparatus (12).

In table 2 the real and imaginary parts are given of the complex shear impedance as found for this solution. Measurements on the same solution were performed in our laboratory with the aid of a torsinal crystal apparatus (12). In order to compare these results with our measurements they were plotted as a function of the reduced frequency $\omega_{R}(18)$. The data quoted in table 2 were obtained by graphical interpolation. The accuracy of these data is estimated to be $\sim 2 \%$.

\section{Discussion}

\subsection{Sensitivity}

The sensitivity of the apparatus compares favourably with other devices working in the same frequency range and is adequate for liquids with a low viscosity. So a liquid with a viscosity of $1 \mathrm{cP}$ shifts the resonance frequency of the fundamental mode some $20 \mathrm{~Hz}$, whereas a shift of $0.5 \mathrm{~Hz}$ is easily detected.

\footnotetext{
$\left.{ }^{3}\right)$ The polystyrene used in this investigation was delivered by Pressure Chemical, Pittsburgh, and had - according to the manufacturer - the following properties: $\bar{M}_{w}=400000, \bar{M}_{w} / \bar{M}_{n}=1.06$; dibutylphthalate, purity $>99 \%$, was obtained from J. T. Baker Chemicals NV. The concentration used was $18.2 \mathrm{mg} / \mathrm{ml}$.
}

\subsection{Reproducibility}

The short term (within \pm 30 minutes) reproducibility is satisfactory; the results of reruns without refilling were within the uncertainty ranges. In order to obtain an idea about the reproducibility over a somewhat longer period various measurements on D.B.P. were repeated after 24 hours. It was found that the deviations of the $3 \mathrm{~dB}$ band-widths were within the uncertainty range of the measurements, but the resonance frequencies showed a definite change. Subsequently two series of measurements were performed on the same tube in air. It was then found that both the differences of the $3 \mathrm{~dB}$ bandwidths and the resonance frequencies between both series were outside the uncertainty range of the measurements. Therefore it must be concluded that the mechanical properties of the tube change with time. Now since the $3 \mathrm{~dB}$ bandwidth of the unloaded tube is small compared to that of the loaded tube, the resulting error in $R_{L}$ is limited. $X_{L}$, however, is determined by the small difference between two almost equal frequencies and a relatively small change in $f_{n}^{0}$ will result in a large error in $X_{L}$. This is probably the cause of the large deviations of $X_{L}$ from its theoretical value in tables 1 and 2. To cope rigourously with this phenomenon it is necessary, in view of the rather time consuming measuring procedure, to calibrate the tube before and after each measurement, as described by Cooke and Matheson (2), and to reject the measurement if the deviation exceeds a certain level. It is clear that this puts a severe limitation on the capability of the apparatus. In order to eliminate this limitation measurements and data handling have to be performed much faster and this calls for a measuring procedure based on the use of an on-line microprocessor, which is now under development (12).

\subsection{Accuracy}

Apart from systematic errors due to changes in the tube material the accuracy of the measured values of the real and imaginary parts of the liquid shear impedance varies from $2-4 \%$. Two error sources are readily located. In the first place the accuracy is limited by the a.c. voltmeter, see figures $3 \mathrm{a}$ and $3 \mathrm{~b}$, for which a high quality digital voltmeter has to be substituted. A second limitation to the accuracy is formed by mode coupling between successive 
overtones, especially when the tube is heavily loaded. This can be overcome by using a tube with a greater wall thickness, but at the cost of sensitivity.

It should now be observed that although only odd overtones are useful, even overtones are excited as well. Elimination of these even overtones would reduce mode coupling to a great extent. This could be achieved by both clamping and exciting the tube in the middle. Attempts are therefore being made to realize such a construction, but so far without succes.

\section{Summary}

An apparatus for the measurement of liquid-shear impedance in the frequency range $4-200 \mathrm{kHz}$ with the aid of a thin-walled Ni-tube resonator is described. A magnetostrictive mechanism is used for setting the tube into torsional oscillation. Real and imaginary parts of the liquid-shear impedance are found from the change in the $3 \mathrm{~dB}$ band-width of the resonance curve and the shift of the resonance frequency, respectively, when the tube is immersed from the air into the liquid. The amount of liquid required is $20 \mathrm{ml}$. The necessary theory is given and some preliminary results are presented.

\section{Zusammenfassung}

Es wird über einen Apparat zur Messung der Scherimpedanz von Flïssigkeiten im Frequenzbereich von 4 bis $200 \mathrm{kHz}$ mit Hilfe eines Resonators berichtet. Der Resonator, ein Nickelrohr mit geringer Wandstärke, wird mittels eines magnetostriktiven Mechanismus in Torsionsschwingungen versetzt. Real- und Imaginärteil der Scherimpedanz der Flüssigkeit werden aus der Änderung der Bandbreite der Resonanzkurve und der Verschiebung der Resonanzfrequenz, wenn das Rohr aus der Luft in die Flüssigkeit eingetaucht wird, berechnet. Die benötigte Flüssigkeitsmenge beträgt $20 \mathrm{ml}$. Die zugehörige Theorie wird mitgeteilt, und einige vorläufige Meßergebnisse werden vorgestellt.

\section{References}

1) Giover, G. M., G. Hail, A. J. Matheson, J. L. Stretton, J. Phys. E 1, 383 (1968).

2) Cooke, B. J., A. J. Matheson, J. Chem. S. F 2 72, 679 (1976).

3) Nakajima, H., H. Okamoto, Y. Wada, Polym. J. 5, 268 (1973).

4) Kolsky, H., Stress Waves in Solids, p. 65, Clarendon (Oxford 1953).

5) McSkimin, H. J., J. Acoust. Soc. Amer. 24, 355 (1952).

6) Waterman, H. A., J. Phys. D 3, 290 (1970).

7) Bariow, A. J., G. Harrison, J. Richter, H. Seguin, J. Lamb, Lab. Pract. 10, 786 (1961).

8) Sakanishi, A., H. Tanaka, Jap. J. Appl. Phys. 12, 1410 (1973).

9) Nederveen, C. J., Internal Report C. L. 68/42, TNO Delft.

10) Drapen, Smith, Applied Regression Analysis, John Wiley \& Sons (1966).

11) Ostle, B., Statistics in Research, Iowa State University Press (1963).

12) Oosterbroek, $M$., et al., to be published.

13) Ferry, J. D., Viscoelastic properties of polymers, Chap. 9, John Wiley \& Sons (New York 1969).

\section{Author's address:}

H. A. Waterman et al.

Twente University of Technology

Department of Applied Physics

Enschede (The Netherlands) 